\title{
DISTRIBUTED KALMAN FILTERING BASED ON QUANTIZED INNOVATIONS
}

\author{
Eric J. Msechu, Alejandro Ribeiro, Stergios I. Roumeliotis, and Georgios B. Giannakis \\ University of Minnesota, 200 Union Street SE, Minneapolis, MN 55455, USA
}

\begin{abstract}
We consider state estimation of a Markov stochastic process using an ad hoc wireless sensor network (WSN) based on noisy linear observations. Due to power and bandwidth constraints present in resourcelimited WSNs, the observations are quantized before transmission. We derive a distributed recursive mean-square error (MSE) optimal quantizer-estimator based on the quantized observations. The resultant Kalman-like algorithm based on quantized observations exhibits MSE performance and computational complexity comparable to the Kalman filter based on un-quantized observations even for 2-3 bits of quantization per observation.
\end{abstract}

Index Terms - wireless sensor networks, distributed state estimation, Kalman filtering, target tracking, limited-rate communication.

\section{INTRODUCTION}

Consider an ad hoc wireless sensor network (WSN) tracking a Markov random process where each sensor acquires noisy observations of a common state. Sensors transmit their observations to each other in order to form a state estimate. If observations were available at a common location, minimum mean-square error (MMSE) estimates could be obtained using a Kalman filter (KF) [1, Chapter 5.5]. However, since observations are distributed in space and there is limited communication bandwidth, the observations are quantized before transmission. The problem is thus transformed into distributed state estimation based on quantized observations.

Quantizing observations to estimate a parameter of interest, is not the same as quantizing a signal for later reconstruction [2]. Instead of a reconstruction algorithm, the objective is finding, e.g., MMSE optimal, estimators using quantized observations. By using binary quantization defined by the sign function, it is possible to derive a filter with complexity and performance close to the clairvoyant KF based on the un-quantized observations [3]. Even though promising, the approach of [3] is limited to a particular 1-bit per observation quantizer.

This paper builds on and considerably broadens the scope of [3] by addressing the middle ground between estimators based on severely quantized (1-bit) data and those based on un-quantized data. The end result is a multi-bit quantized Kalman filter (QKF) that trades bandwidth utilization (dictating the number of quantization bits used for inter-sensor communications) and overall tracking performance (assessed by the mean-square state estimation error).

The rest of the paper is organized as follows: Problem statement and modeling assumptions are presented in Section 2. Section 3 describes the KF based on quantized observations. Simulations in Section 4 are used to corroborate the analytical discourse and compare the

Work in this paper was supported by the USDoD ARO Grant No W911NF-05-1-0283; and also through collaborative participation in the C\&N Consortium sponsored by the U. S. ARL under the CTA Program, Cooperative Agreement DAAD19-01-2-0011. The U. S. Government is authorized to reproduce and distribute reprints for Government purposes notwithstanding any copyright notation thereon. estimator based on quantized data to the clairvoyant KF case based on un-quantized data. Concluding remarks are in Section 5.

Notation: Vectors (resp. matrices) are denoted using lower (upper) case bold face letters. The probability density function (pdf) of $x$ conditioned on $y$ is represented by $p(x \mid y)$. The Gaussian pdf with mean $\mathrm{E}\{\mathbf{x}\}=\boldsymbol{\mu}$ and covariance matrix $\operatorname{cov}\{\mathbf{x}\}=\mathbf{C}$ is represented as $p(\mathbf{x})=\mathcal{N}[\mathbf{x} ; \boldsymbol{\mu}, \mathbf{C}]$ and $Q(z):=\int_{z}^{\infty} \mathcal{N}[x ; 0,1] d x$. The probability mass function for $b$ is denoted as $\operatorname{Pr}\{b\}$. Estimators (or estimates) will be represented using a hat, e.g., $\hat{\mathbf{x}}\left(n \mid \mathbf{w}_{1: m}\right)$, where $\mathbf{w}_{1: m}:=$ $\{w(1), w(2), \ldots w(m)\}$. Finally, ${ }^{T}$ will stand for transposition and $\delta_{i j}($ resp. $\delta(t))$ for the Kronecker (Dirac) delta function.

\section{MODELING AND PROBLEM STATEMENT}

Consider an ad hoc WSN whose $K$ sensor nodes $\left\{\mathcal{S}_{k}\right\}_{k=1}^{K}$ are deployed to estimate a multivariate random process $\mathbf{x}_{c}(t) \in \mathbb{R}^{p}$, where ${ }^{c}$ denotes continuous time. The underlying state equation is

$$
\dot{\mathbf{x}}_{c}(t)=\mathbf{A}_{c}(t) \mathbf{x}_{c}(t)+\mathbf{u}_{c}(t)
$$

where $\mathbf{A}_{c}(t) \in \mathbb{R}^{p \times p}$ denotes the state transition matrix and $\mathbf{u}_{c}(t)$ is a zero-mean white Gaussian noise process with covariance matrix $\mathrm{E}\left\{\mathbf{u}_{c}(t) \mathbf{u}_{c}^{T}(\tau)\right\}=\mathbf{C}_{u_{c}}(t) \delta_{c}(t-\tau)$.

The $k$-th sensor $\mathcal{S}_{k}$ records scalar observations

$$
y_{c, k}(t)=\mathbf{h}_{c, k}^{T}(t) \mathbf{x}_{c}(t)+v_{c, k}(t)
$$

where $\mathbf{h}_{c, k}(t) \in \mathbb{R}^{p}$ denotes the regression vector, and $v_{c, k}(t)$ is a temporally and spatially white zero-mean Gaussian noise process with covariance $\mathrm{E}\left\{v_{c, k}(t) v_{c, l}(\tau)\right\}=c_{v_{c}}(t) \delta(t-\tau) \delta_{k l}$. It is further assumed that $\mathbf{u}_{c}(t)$ is independent of both $v_{c, k}(t)$ and $\mathbf{x}_{c}\left(t_{0}\right)$, where $t_{0}$ is an arbitrary initial reference time.

The discrete-time counterpart of (1) is obtained using the definitions $\boldsymbol{\Phi}\left(t_{2}, t_{1}\right):=\exp \left[\int_{t_{1}}^{t_{2}} \mathbf{A}_{c}(t) d t\right], \mathbf{x}(n):=\mathbf{x}_{c}\left(n T_{s}\right)$, and $\mathbf{u}(n):=\int_{(n-1) T_{s}}^{n T_{s}} \boldsymbol{\Phi}\left(n T_{s}, \tau\right) \mathbf{u}_{c}(\tau) d \tau$, where $T_{s}$ is the sampling period. The discrete-time state equation is [1, Section 4.9]

$$
\mathbf{x}(n)=\mathbf{A}(n) \mathbf{x}(n-1)+\mathbf{u}(n)
$$

where $\mathbf{u}(n)$ is zero-mean white Gaussian noise with covariance matrix $\mathbf{C}_{u}(n)=\int_{(n-1) T_{s}}^{n T_{s}} \boldsymbol{\Phi}\left(n T_{s}, \tau\right) \mathbf{C}_{u_{c}}(\tau) \boldsymbol{\Phi}^{T}\left(n T_{s}, \tau\right) d \tau$ and $\mathbf{A}(n):=$ $\boldsymbol{\Phi}\left(n T_{s},(n-1) T_{s}\right)$. The discrete-time counterpart of the observation equation (2) is given by

$$
y_{k}(n)=\mathbf{h}_{k}^{T}(n) \mathbf{x}(n)+v_{k}(n)
$$

where $y_{k}(n):=y_{c, k}\left(n T_{s}\right)$ is obtained by uniform sampling of $y_{c, k}(t)$ in (2) followed by low- or band-pass filtering with bandwidth $1 / T_{s}$, leading to zero-mean white Gaussian discrete-time noise $v_{k}(n)$ with variance $c_{v}(n)=c_{v_{c}}\left(n T_{s}\right) / T_{s}[1$, Section 4.9].

In order to collaboratively estimate $\mathbf{x}(n)$, each sensor $\mathcal{S}_{k}$ needs to communicate $y_{k}(n)$ to all other sensor nodes $\left\{\mathcal{S}_{l}\right\}_{l=1, l \neq k}^{K}$ by broadcasting over the wireless channel. It is assumed that the sensor scheduling protocol allows for one sensor transmission per time slot in a roundrobin fashion such that $\mathcal{S}_{k}=\mathcal{S}(n)$ is active at time $n$. This leads to a 
one-to-one correspondence between sensor index and time slot, i.e., at time $n, y_{k}(n)=y(n)$. See e.g., [4] and references therein for further discussion on sensor scheduling.

\subsection{MMSE estimation with quantized observations}

In order to effect digital inter-sensor communications in a bandwidthlimited WSN, the observations $y(n) \in \mathbb{R}$ are quantized before broadcasting. With $\mathcal{B}$ denoting a finite set of quantization messages, we define a quantization rule $\mathbf{q}_{n}: \mathbb{R} \rightarrow \mathcal{B}$ so that $b(n):=\mathbf{q}_{n}[y(n)]$. Note that each quantization message, $b(n) \in \mathcal{B}$, can be represented by $\log _{2}|\mathcal{B}|$ bits. Given past and current messages at time $n, \mathbf{b}_{1: n}:=$ $\{b(1), b(2), \ldots, b(n)\}$, the MMSE estimator is given by the conditional mean, see e.g., [1, Chapter 5]

$$
\hat{\mathbf{x}}\left(n \mid \mathbf{b}_{1: n}\right)=\mathrm{E}\left\{\mathbf{x}(n) \mid \mathbf{b}_{1: n}\right\}:=\int_{\mathbb{R}^{p}} \mathbf{x}(n) p\left[\mathbf{x}(n) \mid \mathbf{b}_{1: n}\right] d \mathbf{x}(n) .
$$

In order to obtain a closed-form expression for $\hat{\mathbf{x}}\left(n \mid \mathbf{b}_{1: n}\right)$, the posterior pdf $p\left[\mathbf{x}(n) \mid \mathbf{b}_{1: n}\right]$ has to be known and the integral in (5) needs to be computable. The pdf $p\left[\mathbf{x}(n) \mid \mathbf{b}_{1: n}\right]$ can be, in principle, obtained from the state space model in (3)-(4) using the prediction-correction steps [P1]-[C1] outlined next.

[P1] Prediction step. With $p\left[\mathbf{x}(n-1) \mid \mathbf{b}_{1: n-1}\right]$ known, the theorem of total probability implies that

$$
\begin{aligned}
& p[\left.\mathbf{x}(n) \mid \mathbf{b}_{1: n-1}\right] \\
& \quad=\int_{\mathbb{R}^{p}} p\left[\mathbf{x}(n) \mid \mathbf{x}(n-1), \mathbf{b}_{1: n-1}\right] p\left[\mathbf{x}(n-1) \mid \mathbf{b}_{1: n-1}\right] d \mathbf{x}(n-1) .
\end{aligned}
$$

[C1] Correction step. When quantized observations $b(n)$ become available Bayes' rule allows us to write

$$
p\left[\mathbf{x}(n) \mid \mathbf{b}_{1: n}\right]=p\left[\mathbf{x}(n) \mid \mathbf{b}_{1: n-1}\right] \frac{\operatorname{Pr}\left\{b(n) \mid \mathbf{x}(n), \mathbf{b}_{1: n-1}\right\}}{\operatorname{Pr}\left\{b(n) \mid \mathbf{b}_{1: n-1}\right\}} .
$$

The form of $\operatorname{Pr}\left\{b(n) \mid \mathbf{x}(n), \mathbf{b}_{1: n-1}\right\}$ and $\operatorname{Pr}\left\{b(n) \mid \mathbf{b}_{1: n-1}\right\}$ depend, in part, on the quantization rule $b(n)=\mathbf{q}_{n}[y(n)]$. A well-known example of an iteration of the form [P1]-[C1] corresponds to un-quantized messages $b(n)=y(n)$, i.e., a clairvoyant scenario without bandwidth constraints. In this case, the pdfs in (6) and (7) are Gaussian and by propagating their means and covariance matrices we obtain the clairvoyant Kalman recursions [1, Chapter 5.3]. The pdf in (6) then becomes $\mathcal{N}\left[\mathbf{x}(n) ; \hat{\mathbf{x}}\left(n \mid \mathbf{y}_{1: n-1}\right), \mathbf{M}\left(n \mid \mathbf{y}_{1: n-1}\right)\right]$ with mean and covariance matrix given by

$$
\begin{aligned}
\hat{\mathbf{x}}\left(n \mid \mathbf{y}_{1: n-1}\right) & =\mathbf{A}(n) \hat{\mathbf{x}}\left(n-1 \mid \mathbf{y}_{1: n-1}\right) \\
\mathbf{M}\left(n \mid \mathbf{y}_{1: n-1}\right) & =\mathbf{A}(n) \mathbf{M}\left(n-1 \mid \mathbf{y}_{1: n-1}\right) \mathbf{A}^{T}(n)+\mathbf{C}_{u}(n) .
\end{aligned}
$$

The pdf in (7) is also Gaussian $\mathcal{N}\left[\mathbf{x}(n) ; \hat{\mathbf{x}}\left(n \mid \mathbf{y}_{1: n}\right), \mathbf{M}\left(n \mid \mathbf{y}_{1: n}\right)\right]$. With $\tilde{y}\left(n \mid \mathbf{y}_{1: n-1}\right):=y(n)-\mathbf{h}^{T}(n) \hat{\mathbf{x}}\left(n \mid \mathbf{y}_{1: n-1}\right)$, we have

$$
\begin{aligned}
\hat{\mathbf{x}}\left(n \mid \mathbf{y}_{1: n}\right)= & \hat{\mathbf{x}}\left(n \mid \mathbf{y}_{1: n-1}\right) \\
& +\frac{\mathbf{M}\left(n \mid \mathbf{y}_{1: n-1}\right) \mathbf{h}(n)}{\mathbf{h}^{T}(n) \mathbf{M}\left(n \mid \mathbf{y}_{1: n-1}\right) \mathbf{h}(n)+c_{v}(n)} \tilde{y}\left(n \mid \mathbf{y}_{1: n-1}\right)
\end{aligned}
$$

$$
\begin{aligned}
\mathbf{M}\left(n \mid \mathbf{y}_{1: n}\right)= & \mathbf{M}\left(n \mid \mathbf{y}_{1: n-1}\right) \\
& -\frac{\mathbf{M}\left(n \mid \mathbf{y}_{1: n-1}\right) \mathbf{h}(n) \mathbf{h}^{T}(n) \mathbf{M}\left(n \mid \mathbf{y}_{1: n-1}\right)}{\mathbf{h}^{T}(n) \mathbf{M}\left(n \mid \mathbf{y}_{1: n-1}\right) \mathbf{h}(n)+c_{v}(n)} .
\end{aligned}
$$

The KF iterations in (8) - (11) require a few algebraic operations per time-step $n$ whereas for the iteration [P1]-[C1],with quantized observations, numerical methods are needed to approximate the pdfs in (6) and (7) and to evaluate the expectation in (5)
The objective of this work is to develop an approximate MMSE estimation algorithm based on a Gaussian approximation $p\left[\mathbf{x}(n) \mid \mathbf{b}_{1: n-1}\right]$ $\approx \mathcal{N}\left[\mathbf{x}(n) ; \hat{\mathbf{x}}\left(n \mid \mathbf{b}_{1: n-1}\right), \mathbf{M}\left(n \mid \mathbf{b}_{1: n-1}\right)\right]$. We will see that the proposed algorithm has comparable computational cost to the KF. We will further compare the MSE of the resulting algorithm with that of the clairvoyant KF by quantifying analytically the MSE performance loss due to quantization.

\section{KALMAN FILTER WITH QUANTIZED OBSERVATIONS}

Quantization of $y(n) \in \mathbb{R}$ with $N$-level quantizer is achieved by partitioning $\mathbb{R}$ into non-overlapping intervals $\mathcal{R}_{i}:=\left[\tau_{i}(n), \tau_{i+1}(n)\right)$ where, $i \in \mathcal{B}=\{1, \ldots, N\}$. The quantizer $\mathbf{q}_{n}[\cdot]$ is thus defined by the thresholds $\left\{\tau_{i}(n)\right\}_{i=1}^{N+1}$, where $\tau_{1}(n)=-\infty, \tau_{i}(n)<\tau_{i+1}(n)$, and $\tau_{N+1}(n)=+\infty$. Consider the observation estimate $\hat{y}\left(n \mid \mathbf{b}_{1: n-1}\right)=$ $\mathrm{E}\left\{y(n) \mid \mathbf{b}_{1: n-1}\right\}$, the corresponding innovation $\tilde{y}\left(n \mid \mathbf{b}_{1: n-1}\right):=y(n)-$ $\hat{y}\left(n \mid \mathbf{b}_{1: n-1}\right)$, and the quantization rule

$$
b(n)=i, \quad \text { iff } \quad \tilde{y}\left(n \mid \mathbf{b}_{1: n-1}\right) \in\left[\tau_{i}(n) \tau_{i+1}(n)\right) .
$$

Obtaining an expression for $\hat{\mathbf{x}}\left(n \mid \mathbf{b}_{1: n}\right)$ requires evaluating the integral in (5), which in turn requires the probabilities $\operatorname{Pr}\left\{b(n)=i \mid \mathbf{b}_{1: n-1}\right\}$ and $\operatorname{Pr}\left\{b(n)=i \mid \mathbf{x}(n), \mathbf{b}_{1: n-1}\right\}$ [cf. (7)]. Using the quantization rule in (12) the events $\{b(n)=i\}$ and $\left\{\tau_{i}(n) \leq \tilde{y}\left(n \mid \mathbf{b}_{1: n-1}\right)<\tau_{i+1}(n)\right\}$ are equivalent; and consequently

$$
\begin{aligned}
& \operatorname{Pr}\left\{b(n)=i \mid \mathbf{x}(n), \mathbf{b}_{1: n-1}\right\}= \\
& \operatorname{Pr}\left\{\tau_{i}(n) \leq \tilde{y}\left(n \mid \mathbf{b}_{1: n-1}\right)<\tau_{i+1}(n) \mid \mathbf{x}(n), \mathbf{b}_{1: n-1}\right\} .
\end{aligned}
$$

The innovation $\tilde{y}\left(n \mid \mathbf{b}_{1: n-1}\right):=y(n)-\hat{y}\left(n \mid \mathbf{b}_{1: n-1}\right)=\mathbf{h}^{T}(n)[\mathbf{x}(n)-$ $\left.\hat{\mathbf{x}}\left(n \mid \mathbf{b}_{1: n-1}\right)\right]+v(n)$ has conditional pdf $p\left[\tilde{y}\left(n \mid \mathbf{b}_{1: n-1}\right) \mid \mathbf{x}(n), \mathbf{b}_{1: n-1}\right]$ $=\mathcal{N}\left[\tilde{y}\left(n \mid \mathbf{b}_{1: n-1}\right) ; \mathbf{h}^{T}(n) \tilde{\mathbf{x}}\left(n \mid \mathbf{b}_{1: n-1}\right), c_{v}(n)\right]$, where $\tilde{\mathbf{x}}\left(n \mid \mathbf{b}_{1: n-1}\right)$ $:=\mathbf{x}(n)-\hat{\mathbf{x}}\left(n \mid \mathbf{b}_{1: n-1}\right)$. Using this pdf we can rewrite (13) in terms of the Gaussian tail function $Q[$.$] as$

$\operatorname{Pr}\left\{b(n)=i \mid \mathbf{x}(n), \mathbf{b}_{1: n-1}\right\}$

$=Q\left[\frac{\tau_{i}(n)-\mathbf{h}^{T}(n) \tilde{\mathbf{x}}\left(n \mid \mathbf{b}_{1: n-1}\right)}{\sqrt{c_{v}(n)}}\right]-Q\left[\frac{\tau_{i+1}(n)-\mathbf{h}^{T}(n) \tilde{\mathbf{x}}\left(n \mid \mathbf{b}_{1: n-1}\right)}{\sqrt{c_{v}(n)}}\right]$.

Likewise, we can write the probability $\operatorname{Pr}\left\{b(n)=i \mid \mathbf{b}_{1: n-1}\right\}=$ $\operatorname{Pr}\left\{\tau_{i}(n) \leq \tilde{y}\left(n \mid \mathbf{b}_{1: n-1}\right)<\tau_{i+1}(n) \mid \mathbf{b}_{1: n-1}\right\}$ which is identical to (13) except for the conditioning variables. Note that, unlike (13), the conditional pdf $p\left[\tilde{y}\left(n \mid \mathbf{b}_{1: n-1}\right) \mid \mathbf{b}_{1: n-1}\right]$ is not Gaussian. It can nevertheless be approximated by using a Gaussian pdf for $p\left[\mathbf{x}(n) \mid \mathbf{b}_{1: n-1}\right]$ as follows.

If $p\left[\mathbf{x}(n) \mid \mathbf{b}_{1: n-1}\right]=\mathcal{N}\left[\mathbf{x}(n) ; \hat{\mathbf{x}}\left(n \mid \mathbf{b}_{1: n-1}\right), \mathbf{M}\left(n \mid \mathbf{b}_{1: n-1}\right)\right]$, then the prior pdf $p\left[y(n) \mid \mathbf{b}_{1: n-1}\right]$ is also normal with mean $\hat{y}\left(n \mid \mathbf{b}_{1: n-1}\right)$ and variance $\sigma_{y}^{2}(n):=\mathbf{h}^{T}(n) \mathbf{M}\left(n \mid \mathbf{b}_{1: n-1}\right) \mathbf{h}(n)+c_{v}(n)$. Since the innovation is defined as $\tilde{y}\left(n \mid \mathbf{b}_{1: n-1}\right):=y(n)-\hat{y}\left(n \mid \mathbf{b}_{1: n-1}\right)$, we have $p\left[\tilde{y}\left(n \mid \mathbf{b}_{1: n-1}\right) \mid \mathbf{b}_{1: n-1}\right]=\mathcal{N}\left[\tilde{y}\left(n \mid \mathbf{b}_{1: n-1}\right) ; 0, \sigma_{y}^{2}(n)\right]$ and we can thus write

$$
\begin{aligned}
\operatorname{Pr}\left\{b(n)=i \mid \mathbf{b}_{1: n-1}\right\} & =Q\left[\tau_{i}(n) / \sigma_{y}(n)\right]-Q\left[\tau_{i+1}(n) / \sigma_{y}(n)\right] \\
& =Q\left[\Delta_{i}(n)\right]-Q\left[\Delta_{i+1}(n)\right]
\end{aligned}
$$

where $\Delta_{i}(n):=\tau_{i}(n) / \sigma_{y}(n)$ is a normalized threshold

Substituting (14) and (15) into (7), we obtain an expression for $p\left[\mathbf{x}(n) \mid \mathbf{b}_{1: n}\right]$ that can be used in (5) to obtain $\hat{\mathbf{x}}\left(n \mid \mathbf{b}_{1: n}\right)$. It is remarkable that with the Gaussian assumption for $p\left[\mathbf{x}(n) \mid \mathbf{b}_{1: n-1}\right]$, the integral in (5) can be found analytically as we show in the next proposition (see [5] for proofs of this and subsequent claims). 
Proposition 1 Consider the state space model (3)-(4) and quantized observations $b(n)$ defined in (12). Suppose that the predicted estimate $\hat{\mathbf{x}}\left(n \mid \mathbf{b}_{1: n-1}\right)$ and corresponding error covariance matrix (ECM) $\mathbf{M}\left(n \mid \mathbf{b}_{1: n-1}\right)$ are given. If the predicted $p d f$ is Gaussian $p\left[\mathbf{x}(n) \mid \mathbf{b}_{1: n-1}\right]$ $=\mathcal{N}\left[\mathbf{x}(n) ; \hat{\mathbf{x}}\left(n \mid \mathbf{b}_{1: n-1}\right), \mathbf{M}\left(n \mid \mathbf{b}_{1: n-1}\right)\right]$, the MMSE estimator $\hat{\mathbf{x}}\left(n \mid \mathbf{b}_{1: n}\right)$ in (5) can be obtained as follows:

[C2] Quantized $(\mathbf{Q}) \mathbf{K F}$ correction step. Define the variance of the innovation sequence as $\sigma_{y}^{2}(n):=\mathbf{h}^{T}(n) \mathbf{M}\left(n \mid \mathbf{b}_{1: n-1}\right) \mathbf{h}(n)+c_{v}(n)$ and normalized thresholds $\Delta_{i}(n):=\tau_{i}(n) / \sigma_{y}(n)$. For $b(n)=i$, define the ratios

$$
\begin{aligned}
\alpha_{i}(n) & :=\frac{\mathrm{E}\left\{\tilde{y}\left(n \mid \mathbf{b}_{1: n-1}\right) \mid \mathbf{b}_{1: n-1}, b(n)=i\right\}}{\sigma_{y}(n)} \\
& =\frac{1}{\sqrt{2 \pi}} \frac{e^{-\Delta_{i}^{2}(n) / 2}-e^{-\Delta_{i+1}^{2}(n) / 2}}{Q\left[\Delta_{i}(n)\right]-Q\left[\Delta_{i+1}(n)\right]} \\
\beta_{i}(n) & :=1-\frac{\operatorname{var}\left\{\tilde{y}\left(n \mid \mathbf{b}_{1: n-1}\right) \mid \mathbf{b}_{1: n-1}, b(n)=i\right\}}{\sigma_{y}^{2}(n)} \\
& =\alpha_{i}^{2}(n)-\frac{1}{\sqrt{2 \pi}} \frac{\Delta_{i}(n) e^{-\Delta_{i}^{2}(n) / 2}-\Delta_{i+1}(n) e^{-\Delta_{i+1}^{2}(n) / 2}}{Q\left[\Delta_{i}(n)\right]-Q\left[\Delta_{i+1}(n)\right]} .
\end{aligned}
$$

Then the estimate of $\mathbf{x}(n)$ and its corresponding ECM based on quantized messages are given by

$$
\begin{aligned}
\hat{\mathbf{x}}\left(n \mid \mathbf{b}_{1: n}\right)= & \hat{\mathbf{x}}\left(n \mid \mathbf{b}_{1: n-1}\right) \\
& +\alpha_{i}(n) \frac{\mathbf{M}\left(n \mid \mathbf{b}_{1: n-1}\right) \mathbf{h}(n)}{\sqrt{\mathbf{h}^{T}(n) \mathbf{M}\left(n \mid \mathbf{b}_{1: n-1}\right) \mathbf{h}(n)+c_{v}(n)}} \\
\mathbf{M}\left(n \mid \mathbf{b}_{1: n}\right)= & \mathbf{M}\left(n \mid \mathbf{b}_{1: n-1}\right) \\
& -\beta_{i}(n) \frac{\mathbf{M}\left(n \mid \mathbf{b}_{1: n-1}\right) \mathbf{h}(n) \mathbf{h}^{T}(n) \mathbf{M}\left(n \mid \mathbf{b}_{1: n-1}\right)}{\mathbf{h}^{T}(n) \mathbf{M}\left(n \mid \mathbf{b}_{1: n-1}\right) \mathbf{h}(n)+c_{v}(n)} .
\end{aligned}
$$

Proposition 1 suggests an algorithm for finding (approximate) MMSE state estimates using quantized observations. The resulting QKF consists of recursive application of the prediction step (8)-(9), with $\mathbf{y}_{1: n-1}$ replaced by $\mathbf{b}_{1: n-1}$, and the correction step [C2]

The QKF retains a notable resemblance to the clairvoyant KF (8)(11). Except for the $\beta_{i}(n)$ factor, the ECM for the QKF in (19) has the same form as the ECM for the KF in (11). To compare the state updates (10) of the KF and (18) of the QKF, rewrite (18) as

$$
\begin{aligned}
\hat{\mathbf{x}}\left(n \mid \mathbf{b}_{1: n}\right) & =\hat{\mathbf{x}}\left(n \mid \mathbf{b}_{1: n-1}\right) \\
& +\frac{\mathbf{M}\left(n \mid \mathbf{b}_{1: n-1}\right) \mathbf{h}(n)}{\mathbf{h}^{T}(n) \mathbf{M}\left(n \mid \mathbf{b}_{1: n-1}\right) \mathbf{h}(n)+c_{v}(n)} \tilde{\alpha}_{i}\left(n \mid \mathbf{b}_{1: n-1}\right)
\end{aligned}
$$

where, $\tilde{\alpha}_{i}\left(n \mid \mathbf{b}_{1: n-1}\right):=\sqrt{\mathbf{h}^{T}(n) \mathbf{M}\left(n \mid \mathbf{b}_{1: n-1}\right) \mathbf{h}(n)+c_{v}(n)} \alpha_{i}(n)$ is the QKF innovation. Note that (20) has the same form as the KF correction (10) with $\tilde{\alpha}_{i}\left(n \mid \mathbf{b}_{1: n-1}\right)$ viewed as the QKF innovation counterpart of the KF innovation $\tilde{y}_{i}\left(n \mid \mathbf{y}_{1: n-1}\right)$.

Remark 1 MSE performance of the QKF can be compared with MSE performance of the KF given the same past observations $\left\{\mathbf{b}_{1: n-1}\right\}$. For that matter, define the ECM reduction per correction step, at time $n$, for the QKF as [cf. (19)]

$$
\begin{aligned}
\Delta \mathbf{M}(n): & =\mathbf{M}\left(n \mid \mathbf{b}_{1: n-1}\right)-\mathbf{M}\left(n \mid \mathbf{b}_{1: n}\right) \\
& =\beta_{i}(n) \frac{\mathbf{M}\left(n \mid \mathbf{b}_{1: n-1}\right) \mathbf{h}(n) \mathbf{h}^{T}(n) \mathbf{M}\left(n \mid \mathbf{b}_{1: n-1}\right)}{\mathbf{h}^{T}(n) \mathbf{M}\left(n \mid \mathbf{b}_{1: n-1}\right) \mathbf{h}(n)+c_{v}(n)} .
\end{aligned}
$$

Table 1. $\bar{\beta}(n)$ values for $m=\log _{2} N$ number of bits

\begin{tabular}{|c|l|l|l|l|}
\hline $\mathrm{N}(m$ bits $)$ & $2(1$ bit $)$ & $4(2$ bits $)$ & $8(3$ bits $)$ & $16(4$ bits $)$ \\
\hline$\beta(n)$ & $0.637=2 / \pi$ & 0.883 & 0.966 & 0.991 \\
\hline
\end{tabular}

Equivalently, if we use $b(n)=y(n)$ the corresponding ECM reduction for the KF is [cf. (11)]

$$
\begin{aligned}
\Delta \mathbf{M}^{\mathrm{K}}(n): & =\mathbf{M}\left(n \mid \mathbf{b}_{1: n-1}\right)-\mathbf{M}\left(n \mid \mathbf{b}_{1: n-1}, y(n)\right) \\
& =\frac{\mathbf{M}\left(n \mid \mathbf{b}_{1: n-1}\right) \mathbf{h}(n) \mathbf{h}^{T}(n) \mathbf{M}\left(n \mid \mathbf{b}_{1: n-1}\right)}{\mathbf{h}^{T}(n) \mathbf{M}\left(n \mid \mathbf{b}_{1: n-1}\right) \mathbf{h}(n)+c_{v}(n)} .
\end{aligned}
$$

Comparing (21) to (22), we obtain $\Delta \mathrm{M}(n)=\beta_{i}(n) \Delta \mathrm{M}^{\mathrm{K}}(n)$. That is, the ECM reduction achieved by the QKF in one correction step, is $\beta_{i}(n)$ times the ECM reduction of the clairvoyant KF. From (17) we see that $0<\beta_{i}(n)<1$, consistent with the fact that $b(n)$ contains less information about $\mathbf{x}(n)$ than $y(n)$.

\subsection{Quantizer design}

Quantization in (12) is defined by the quantizer thresholds $\left\{\tau_{i}(n)\right\}_{i=1}^{N+1}$. The ECM $\mathbf{M}\left(n \mid \mathbf{b}_{1: n}\right)$ in (19), and consequently the ECM reduction $\Delta \mathbf{M}(n)$ in (21), depend on $b(n)$. We define the optimal quantizer as the one that maximizes the average variance reduction, i.e.,

$$
\left\{\Delta_{i}^{*}(n)\right\}_{i=2}^{N}:=\underset{\left\{\Delta_{i}(n)\right\}_{i=2}^{N}}{\arg \max _{b(n)}}\left\{\Delta \mathbf{M}(n) \mid \mathbf{b}_{1: n-1}\right\} .
$$

It is readily verified that $\left\{\Delta_{i}^{*}(n)\right\}_{i=2}^{N}$ in (23) can be obtained as the maximizers of $\bar{\beta}(n):=\mathrm{E}_{b(n)}\left\{\beta_{i}(n) \mid \mathbf{b}_{1: n-1}\right\}$. Using the expression for $\operatorname{Pr}\left\{b(n)=i \mid \mathbf{b}_{1: n-1}\right\}$ in (15), $\bar{\beta}(n)$ can be written as

$$
\begin{aligned}
\bar{\beta}(n) & =\sum_{i=1}^{N}\left[Q\left[\Delta_{i}(n)\right]-Q\left[\Delta_{i+1}(n)\right]\right] \beta_{i}(n) \\
& =\frac{1}{2 \pi} \sum_{i=1}^{N} \frac{\left[\exp \left(-\Delta_{i}^{2}(n) / 2\right)-\exp \left(-\Delta_{i+1}^{2}(n) / 2\right)\right]^{2}}{Q\left[\Delta_{i}(n)\right]-Q\left[\Delta_{i+1}(n)\right]}
\end{aligned}
$$

where in the second equality we used the definitions of $\alpha_{i}(n)$ in (16) and $\beta_{i}(n)$ in (17). Using (24) we can obtain the following proposition.

Proposition 2 Consider the problem of optimal quantization of the innovation $\tilde{y}\left(n \mid \mathbf{b}_{1: n-1}\right)$. If $b(n)=i$, the reconstructed innovation is $\hat{\tilde{y}}^{(i)}\left(n \mid \mathbf{b}_{1: n-1}\right):=\mathrm{E}\left\{\tilde{y}\left(n \mid \mathbf{b}_{1: n-1}\right) \mid \mathbf{b}_{1: n-1}, b(n)=i\right\}=\sigma_{y}(n) \alpha_{i}(n)$ [cf. (16)]. Using an MSE distortion, the optimal quantizer of the innovation $\tilde{y}\left(n \mid \mathbf{b}_{1: n-1}\right)$ is given as

$$
\begin{aligned}
& \left\{\Delta_{i}^{\dagger}(n)\right\}_{i=2}^{N} \\
& :=\arg \min _{\left\{\Delta_{i}(n)\right\}_{i=2}^{N}} \mathrm{E}\left\{\left[\tilde{y}\left(n \mid \mathbf{b}_{1: n-1}\right)-\hat{\tilde{y}}^{(i)}\left(n \mid \mathbf{b}_{1: n-1}\right)\right]^{2} \mid \mathbf{b}_{1: n-1}\right\} .
\end{aligned}
$$

The optimal thresholds in (23) and (25) are equal, i.e., $\left\{\Delta_{i}^{*}(n)\right\}_{i=2}^{N}=$ $\left\{\Delta_{i}^{\dagger}(n)\right\}_{i=2}^{N}$.

Proposition 2 asserts that the optimal strategy for quantizing the innovations $\tilde{y}\left(n \mid \mathbf{b}_{1: n-1}\right)$ in order to estimate $\hat{\mathbf{x}}\left(n \mid \mathbf{b}_{1: n}\right)$ is to quantize $\tilde{y}\left(n \mid \mathbf{b}_{1: n-1}\right)$ with minimum MSE distortion.

Since $p\left[\tilde{y}\left(n \mid \mathbf{b}_{1: n-1}\right) \mid \mathbf{b}_{1: n-1}\right] \approx \mathcal{N}\left[\tilde{y}\left(n \mid \mathbf{b}_{1: n-1}\right) ; 0, \sigma_{y_{n}}^{2}\right]$, the optimal MSE quantizer for $\tilde{y}\left(n \mid \mathbf{b}_{1: n-1}\right)$ is given by the Lloyd-Max quantizer of [6]. The optimal normalized thresholds values, i.e., $\left\{\Delta_{i}^{\dagger}(n)\right\}_{i=1}^{N+1}$, from [6] are used in (24) and the calculated $\bar{\beta}(n)$ values are summarized in Table 1. Note that quantizing to more than 4 bits, for which $\bar{\beta}(n) \approx 0.99$, seems rather unjustified. In the next section, simulations for the QKF are provided in order to corroborate the analytical statements and validate model consistency. 


\section{SIMULATIONS}

The two-dimensional state space model simulated is

$$
\dot{\mathbf{x}}_{c}(t):=\left(\begin{array}{l}
\dot{x}_{c_{1}}(t) \\
\dot{x}_{c_{2}}(t)
\end{array}\right)=\left(\begin{array}{ll}
0 & 1 \\
0 & 0
\end{array}\right)\left(\begin{array}{l}
x_{c_{1}}(t) \\
x_{c_{2}}(t)
\end{array}\right)+\left(\begin{array}{l}
0 \\
1
\end{array}\right) u_{c}(t)
$$

This model is known as constant-velocity tracking model in [7, pp. 82]. Noisy measurements at sensor $\mathcal{S}_{k}$ are given as $y_{k}(t)=x_{c_{1}}(t)+$ $\theta_{k} x_{c_{2}}(t)+v_{k}(t)$, where $\theta_{k}$ is a parameter at sensor $k$. A discretetime equivalent model is used with sampling period $T_{s}=1$. Simulations were performed for $K=2$ sensors with $\left[\begin{array}{ll}\theta_{1} & \theta_{2}\end{array}\right]=\left[\begin{array}{ll}0.1 & 0.2\end{array}\right]$, $c_{v}(t)=1$ and $c_{u}(t)=1$.

In Fig. 1 QKF MSE, i.e., $\operatorname{tr}\left\{\mathbf{M}\left(n \mid \mathbf{b}_{1: n}\right)\right\}$, for 1,2 and 3 bits is compared with the MSE of the clairvoyant KF. The plots demonstrate the MSE improvement offered by 2 bits of quantization compared to the 1-bit QKF case. Quantizing with more than 2 bits offers little MSE improvement.

Model consistency tests comparing the empirical MSEs with analytical MSEs are depicted in Fig. 2. The analytical MSEs, as defined in (9) and (19) are compared with empirically computed MSEs (sample average of the squared estimation errors) for both predictor MSE and estimator MSE. This consistency test reveals that the empirical and analytical MSEs are nearly identical.

Fig. 3 shows the normalized estimation error squared (NEES) consistency test statistics for the QKF [7, Chapter 5.4]. With $L$ realizations of the NEES statistics $\left\{r_{i}(n)\right\}_{i=1}^{L}$, where $r(n):=[\mathbf{x}(n)-$ $\left.\hat{\mathbf{x}}\left(n \mid \mathbf{b}_{1: n}\right)\right]^{T}\left[\mathbf{M}\left(n \mid \mathbf{b}_{1: n}\right)\right]^{-1}\left[\mathbf{x}(n)-\hat{\mathbf{x}}\left(n \mid \mathbf{b}_{1: n}\right)\right]$ and using $\hat{r}(n):=$ $\frac{1}{L} \sum_{i=1}^{L} r_{i}(n)$, an acceptance (confidence) region $\operatorname{Pr}\{\hat{r}(n) \in[l, u]\}=$ $1-\alpha$, is defined. From $L=100$ realizations, $p=2$ state dimensions, and $\alpha=0.05$ (i.e., $95 \%$ region), we observed that indeed only $5 \%$ of the 100 time samples were outside the $95 \%$ acceptance region.

\section{CONCLUDING REMARKS}

A distributed recursive state estimator based on quantized observations was developed for tracking Markov state space models. It was shown both analytically and in simulation that quantizing using $2-3$ bits improves the mean-square error performance of the Kalman-like algorithm to virtually coincide with the optimal state estimator (Kalman filter), with only minimal increase in computation. It was further shown that actual state estimation MSEs are consistent with the estimator algorithm's MSEs.

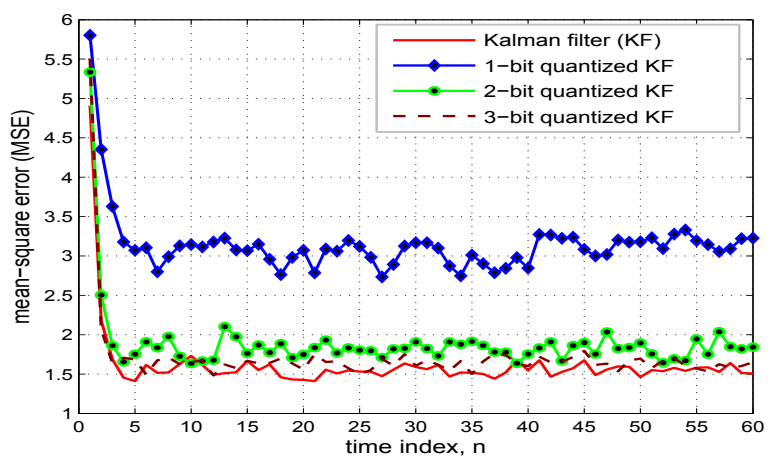

Fig. 1. MSEs for 1-3 bits of QKF and MSE for KF.

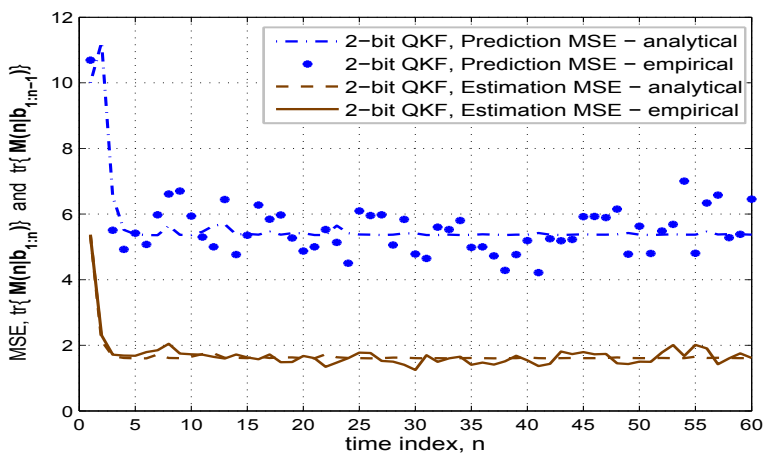

Fig. 2. Analytical vs. Empirical MSEs.

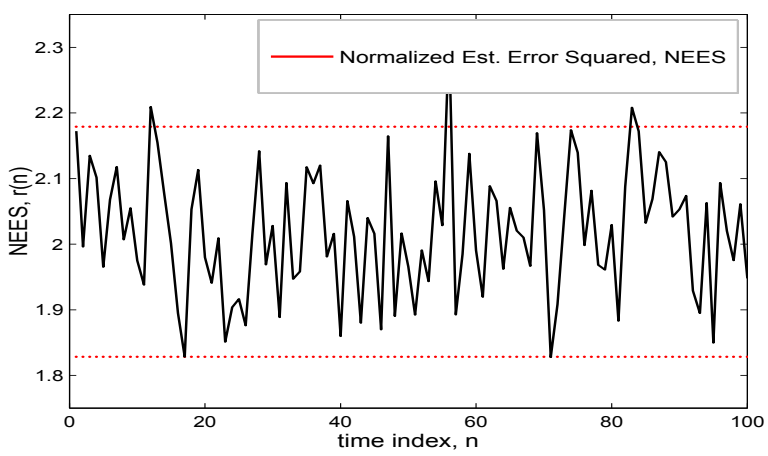

Fig. 3. NEES consistency test.

\section{REFERENCES}

[1] P. S. Maybeck, Stochastic Models, Estimation and Control - Vol.1, Academic Press, 1979.

[2] R. M. Gray, "Quantization in task-driven sensing and distributed processing," in Proc. Int. Conf. Acoustics, Speech, Sig. Proc., Toulouse, France, vol. 5, pp. V-1049 - V-1052, May 14-19, 2006.

[3] A. Ribeiro, G. B. Giannakis, and S. I. Roumeliotis, "SOI-KF: Distributed Kalman filtering with low-cost communications using the sign of innovations," IEEE Transactions on Signal Processing, vol. 54, no. 12, pp. 4782-4795, Dec 2006.

[4] V. Gupta, T. Chung, B. Hassibi, and R.M. Murray, "Sensor scheduling algorithms requiring limited computation," in Proc. Int. Conf. Acoustics, Speech, Signal Processing, Montreal, Canada, vol. 3, pp. iii-825 - iii-828, 17-21 May, 2004.

[5] E. J. Msechu, S. I. Roumeliotis, A. Ribeiro, and G. B. Giannakis, "Distributed quantized Kalman filtering with scalable communication cost," IEEE Trans. on Sig. Proc., Aug. 2007 (submitted) available at http://spincom.ece.umn.edu/journal.html.

[6] J. Max, "Quantizing for minimum distortion," IEEE Trans. on Information Theory, vol. 6, no. 1, pp. 7-12, Mar. 1960.

[7] Y. Bar-Shalom and X. Li, Estimation and Tracking: Principles, Techniques, and Software, Artech House, 1993. 